\title{
Drug discovery and development for neglected diseases: the DNDi model
}

This article was published in the following Dove Press journal:

Drug Design, Development and Therapy

I5 March 201 I

Number of times this article has been viewed

\author{
Eric Chatelain \\ Jean-Robert loset \\ Drugs for Neglected Diseases \\ Initiative (DNDi), Geneva, \\ Switzerland
}

Correspondence: Jean-Robert loset DNDi, I5 Chemin Louis Dunant, $\mathrm{CH}-\mathrm{I} 202$ Geneva, Switzerland $\mathrm{Tel}+4$ I 229069230 Fax +4I 229069231 Email jrioset@dndi.org

\begin{abstract}
New models of drug discovery have been developed to overcome the lack of modern and effective drugs for neglected diseases such as human African trypanosomiasis (HAT; sleeping sickness), leishmaniasis, and Chagas disease, which have no financial viability for the pharmaceutical industry. With the purpose of combining the skills and research capacity in academia, pharmaceutical industry, and contract researchers, public-private partnerships or product development partnerships aim to create focused research consortia that address all aspects of drug discovery and development. These consortia not only emulate the projects within pharmaceutical and biotechnology industries, eg, identification and screening of libraries, medicinal chemistry, pharmacology and pharmacodynamics, formulation development, and manufacturing, but also use and strengthen existing capacity in disease-endemic countries, particularly for the conduct of clinical trials. The Drugs for Neglected Diseases initiative (DNDi) has adopted a model closely related to that of a virtual biotechnology company for the identification and optimization of drug leads. The application of this model to the development of drug candidates for the kinetoplastid infections of HAT, Chagas disease, and leishmaniasis has already led to the identification of new candidates issued from DND $i$ 's own discovery pipeline. This demonstrates that the model DND $i$ has been implementing is working but its DND $i$, neglected diseases sustainability remains to be proven.
\end{abstract}

Keywords: R\&D, screening, lead optimization, human African trypanosomiasis, leishmaniasis, Chagas disease, product development partnerships

\section{Introduction}

Neglected tropical diseases (NTDs), as defined by the World Health Organization (WHO), refer to infectious diseases that occur within the tropical belt and include - but are not limited to - malaria, leishmaniasis, schistosomiasis, onchocerciasis, lymphatic filariasis, Chagas disease, African trypanosomiasis, and dengue. ${ }^{1-4}$ These diseases are responsible for substantial morbidity and mortality in the developing world and have a clear economic burden on the affected countries. ${ }^{5,6}$ During 1975-2004, only 21 (1.3\%) out of 1556 approved drugs were specifically developed to address NTDs, even though NTDs account for $11.4 \%$ of the global disease burden. ${ }^{7}$ The lack of a profitable market and effective mechanisms related to public health policy, financing, and drug discovery and development expertise and capacity has largely contributed to this low achievement. ${ }^{8}$ Yet, the landscape has dramatically changed in the past 5 years. ${ }^{9}$ Indeed, the emergence of product development partnerships (PDPs) is filling the existing research and development (R\&D) gap and creating bridges between the various private and public stakeholders. ${ }^{10,11}$

The immediate needs of the most neglected patients affected by NTDs are, however, still largely unmet. A recent study by G-FINDER ${ }^{12}$ has revealed that less than 
$5 \%$ of worldwide R\&D funding for NTDs has been directed towards the most neglected diseases, such as human African trypanosomiasis (HAT), visceral leishmaniasis, and Chagas disease, although more than 500 million people are at risk. ${ }^{13}$ By building partnerships in both the public and the private sector, based on existing capacity, expertise, and resources, $\mathrm{DND} i$ is working to foster R\&D for NTDs that continue to affect millions of people worldwide.

\section{DNDi: the organization and its objectives}

$\mathrm{DND} i$ is a not-for-profit drug R\&D organization based in Geneva, Switzerland, founded in 2003 by seven institutions: the Indian Council for Medical Research (ICMR), the Kenya Medical Research Institute (KEMRI), the Malaysian Ministry of Health, the Oswaldo Cruz Foundation in Brazil, Médecins Sans Frontieres (MSF), the Institut Pasteur in France, and the Special Programme for Research and Training in Tropical Diseases (TDR). The aim was to create an organization that would respond to the dire need of safe, affordable, easy-to-use and efficacious treatments for neglected patients. ${ }^{14}$

DNDi's main short-term objectives are to deliver 6-8 new treatments for HAT, Chagas disease, visceral leishmaniasis, and malaria by 2014 and, at the same time, to address the immediate needs of affected patients with a sense of urgency. $\mathrm{DND} i$ also aims to establish a robust $\mathrm{R} \& \mathrm{D}$ portfolio that covers the entire drug discovery process, from early-stage discovery to clinical development and distribution, to respond to patient needs for better, effective, safe, adequate, and accessible new treatments.
Moreover, DND $i$ aims to use and strengthen existing capacities in disease-endemic countries and to raise awareness about the need to develop new drugs for NTDs and advocate for increased public responsibility.

\section{DNDi: R\&D strategy}

To achieve its ambitious goals, DND $i$ has built a solid and well balanced portfolio that spans the entire drug development pipeline for kinetoplastid diseases (Figure 1). With a strategic approach to identify and bridge the gaps across the drug development pipeline, DND $i$ has undertaken both middle/short-term and long-term projects. Short- and medium-term projects aim to deliver new treatments by 2014 . Long-term projects aim to establish a solid pipeline for new drugs after 2014.

DND $i$ builds its portfolio by identifying projects that fall into the following five categories, based on the nature of the compound/treatment under consideration and according to its stage of development or expected time to reach patients:

- New drug candidates identified through screening and lead optimization efforts

- New molecules associated with an advanced development profile - the so-called "low-hanging fruits" or "oldies" (could start at lead optimization or pre-clinical development)

- New indications for existing medicines in the field of the most neglected diseases (ie, therapeutic switching, drug repositioning)

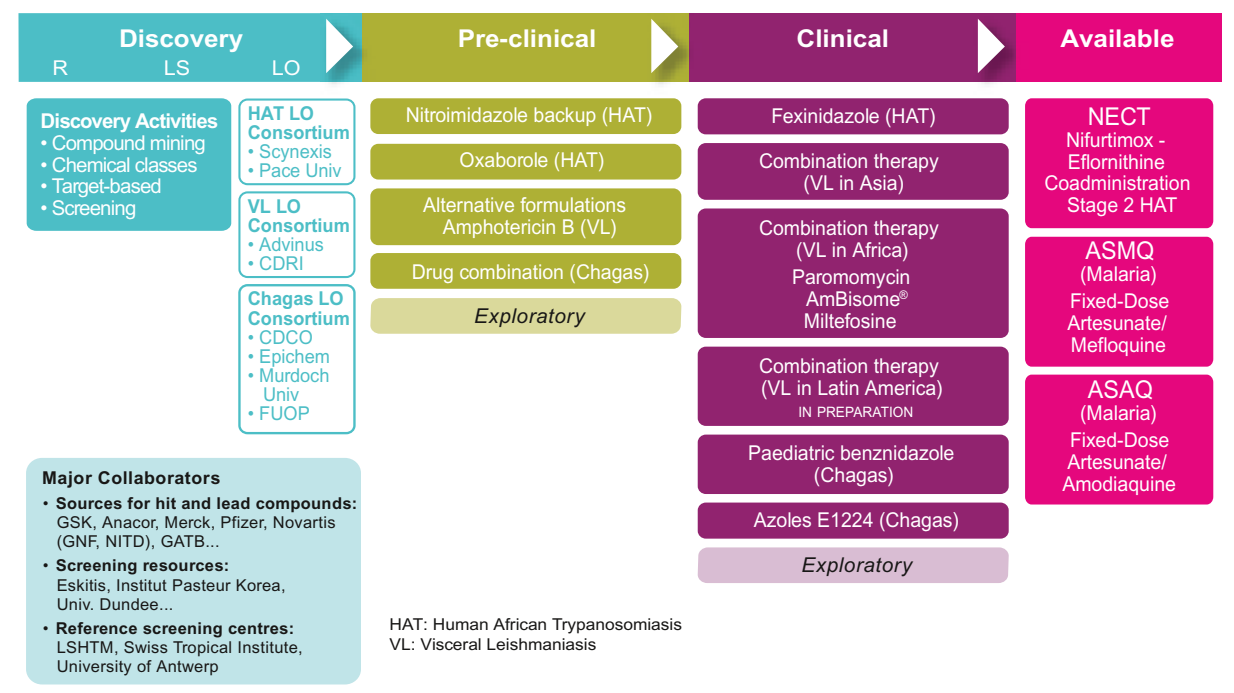

Figure I DNDi portfolio as of January 2010.

Abbreviations: R: research, LS: lead selection, LO: lead optimisation, CDRI: Central Drug Research Institute (India), CDCO: Centre for Drug Candidate Optimisation (Australia), FUOP: Federal University of Ouro Preto (Brazil), GNF: Genomics Institute of the Novartis Research Foundation, NITD: Novartis Institute for Tropical Diseases, GATB: Global Alliance for TB Drug Development, LSHTM: London School of Hygiene \& Tropical Medicine. 
- New formulations and combinations of existing drugs better adapted to field conditions (ie, pediatric, long-acting, new route of administration, fixed-dose combinations, copackaging or coadministration)

- Existing drugs for target diseases (ie, geographical extension of registration, completion of regulatory dossiers of existing drug candidates)

\section{DNDi: Discovery and the "S" rule}

DND $i$ has adopted a pragmatic strategy towards drug discovery that has evolved from a hunter-gatherer approach identifying projects mostly based on networking interactions to structured partnerships with pharmaceutical and biotechnology companies. The partnerships include Sourcing and Screening of compounds followed by the Selection of Series to optimize.

\section{Sourcing}

Because of limitations in screening throughput and a sense of urgency to develop modern medicines for these neglected diseases, DND $i$ has adopted a focused approach to compound sourcing and drug discovery rather than seeking to address chemical diversity through random compound library screening.

Examples of this focused approach for different stages of drug discovery are listed below.

- Chemical diversity: access to diversity sets representative of libraries from various institutions and pharmaceutical companies; natural products, synthetic compounds, random screening.

- Focused drug classes:

- Chemical series: accessed mostly through partnership with pharmaceutical and biotechnology companies with the goal of not only accessing compounds of interest but also libraries of analogs, as well as related pharmaceutical knowledge and expertise associated with those series (eg, oxaboroles at Anacor, pyridones at GSK, and macrolides are examples of chemical classes under development at DNDi); biologically annotated series accelerate over clusters characterized only in terms of potency from a library screen.

- Classes of inhibitors: inhibitors of specific biochemical pathways are evaluated for their activity against efficacy models with the idea to access library of analogs as well as related pharmaceutical knowledge and expertise associated with those series (eg, cysteine protease inhibitors at Merck, partnership with University of Dundee's Drug Discovery Unit).
- Data mining/low-hanging-fruit approach: the aim is to identify compounds reasonably well characterized from a development standpoint, enabling a gain of time and resources in terms of potential development provided that the profile of such molecules is in line with our R\&D criteria. Fexinidazole, a nitroimidazole compound currently in phase I clinical development for HAT at DND $i$ is a good illustration of this approach. ${ }^{15}$

- The DND $i$ discovery team has also developed strong relationships with other PDPs such as the Medicines for Malaria Venture (MMV), the Global Alliance for TB (TB Alliance), as well as with additional R\&D industrial institutes/centers active in the field, such as the Novartis Institute for Tropical Diseases. The objectives of these alliances and/or with other PDPs gravitate around exchange of information and experience, identification of potential synergies, and avoidance of duplication.

Needless to say, the choice of the partner and their commitment to neglected diseases is of crucial importance to move forward quickly in the process. Indeed, access to compounds, but more importantly the knowledge that the partner has for a compound class, can have a dramatic impact on the need for resources, the pace of the discovery process, and nomination of a candidate.

\section{Screening}

In contrast to malaria research in which drug targets have been identified, validated targets are still very sparse or missing for kinetoplastid diseases. For that reason and since discrepancy between target and whole-cell screens have been widely observed in the frame of several antiinfective screening campaigns, DND $i$ took advantage of phenotypic screens to identify active compounds against parasites. Indeed, there are no regulatory requirements to identify the target of an active compound or its mode of action, even if the latter could be helpful during the next steps of development.

Historically, the lack of high throughput capacity of phenotypic screening assays for kinetoplastid diseases has prevented the access to a critical mass of quality hits needed for further development considering the high attrition rate occurring in discovery programs. ${ }^{16}$ This is especially true for intracellular obligate protozoa such as Leishmania donovani and Trypanosoma cruzi (the causal agents of visceral leishmaniasis and Chagas disease, respectively). To address this, DND $i$ has invested considerable effort to develop high-throughput screening (HTS) 
assays for all three diseases of interest; in particular, an HTS image screening assay, developed in partnership with the Institut Pasteur Korea (IPK) and available for the relevant intracellular stage of $L$. donovani, is a key milestone in the field and is expected to change dramatically the hit generation dynamics for visceral leishmaniasis. Just recently, the first ever HTS campaign against the intracellular form of $L$. donovani has been completed at IPK on a library of 200,000 compounds, a number that is most probably larger than the total amount of compounds ever tested against Leishmania so far.

Besides HTS assays, DND $i$, through its network of partners with well established and recognized centers in parasitology, such as the Swiss Tropical Institute and the University of Antwerp among others, is able to evaluate and confirm activities of compounds issued from HTS campaigns with state of the art, low throughput assays both in vitro and in vivo.

The phenotypic screen of smaller specific subsets of compounds belonging to a certain chemical class or target inhibitor could lead to the identification and chemical validation of targets of interest, making target-based screens more attractive in the field of kinetoplastid drug discovery. One such target, the parasite phosphodiesterase that is essential for Trypanosome brucei and L. donovani, is currently being investigated in a collaborative project involving, among others, Nycomed, DND $i$, and TI Pharma.

\section{Series to optimize}

$\mathrm{DND} i$ strives to maintain a full pipeline, which includes development of innovative new drugs through long-term lead optimization programs. Drug candidate series of interest identified from various screening projects are selected to enter the lead optimization process.

For that purpose and because of resources and costs, DND $i$ has created a unique model for lead optimization. In short, DND $i$ has adopted the approach of contracting lead optimization teams that are independent from specific targets or scaffolds. In doing so, DND $i$ can feed each team with hits and leads that are identified from a range of different sources. Scaffolds that prove not to be amenable to optimization can also be discontinued without disrupting the research integrity of the dedicated lead optimization team.

To maintain the capacity for optimizing one lead series at all times, each team consists of five or six chemists, two or three pharmacologists, and a dedicated screening facility to assess potency and efficacy with guaranteed infrastructure to support medicinal chemistry, in vitro and in vivo distributionmetabolism-pharmacokinetic (DMPK), toxicology, and efficacy studies.

To produce new drug candidates as efficiently as possible, all players should be focused on the lead optimization program and not distracted by other research efforts. To that end:

- Once a lead is introduced into a lead optimization program, it enters a critical path ${ }^{17}$ that promises development to patient access unless the compound series fails because structural liabilities prevent optimization as a drug candidate

- Sufficient resources are allocated to guarantee the rapid turnaround of data necessary to support the medicinal chemistry effort

- A research group associated with the program is encouraged to pursue more discovery research associated with the disease so that the lead optimization programs are constantly fed with promising leads. Therefore, a separate group of staff conducts the supporting assays for lead optimization and commits $100 \%$ of its time to the medicinal chemistry effort.

To date, DND $i$ has brought together four lead optimization teams, two for HAT, one for visceral leishmaniasis, and one for Chagas. DND $i$ works with companies and partners that are committed to research for NTDs. Researchers are funded as fee-for-service contractors and make no claim to any intellectual property generated during the lead optimization programs. Therefore, DND $i$ is free to manage the intellectual property in a way that is most appropriate to guarantee access for those affected by NTDs.

\section{Support/selection of the candidate}

To prioritize and select compounds and series, and advance them through the drug development process, DND $i$ has developed a set of tools and consulting committees to support its decision-making process. ${ }^{18}$ These include target product profiles (TPPs) for each disease for both new chemical entities (NCEs) and combination treatments, drug discovery manuals, decision matrices, Web-based data management, and a network of consultants to act as critics and mentors.

Precise and concrete criteria have also been compiled at the preclinical stage to define profiles of hits, leads, optimized leads, and drug preclinical candidates for HAT, visceral leishmaniasis, and Chagas disease. These criteria typically including in vitro and in vivo efficacy data, chemical features such as compound tractability and druggability, absorption-distribution-metabolism-excretion (ADME), 
and toxicity parameters - are described in the DND $i$ Drug Discovery and Development Manual.

\section{Target product profiles}

TPPs are an organized list that prioritizes key features of the drug and guides investigators to work in an orchestrated way. ${ }^{19}$ Therefore, TPPs are the basis of all drug discovery management tools designed to support lead optimization programs.

$\mathrm{DND} i$ is a needs-driven organization (ie, the needs of patients suffering from NTDs are the driving force for all drug discovery and development programs). DNDi, through consultation with expert committees that include stakeholders such as physicians, local disease control programs, regulators, and patient representatives, as well as local and global public health organizations, is able to draft TPPs. Therefore, TPPs define effective therapies for patients in every-day field conditions (see Table 1 for an example of TPP).

\section{Discovery manuals}

To direct progression of compound during the drug discovery process, drug discovery manuals for each disease are prepared to define key decision points and provide objective measures for making decisions, taking into account potency, physicochemical properties and in vitro and in vivo DMPK/ toxicity. Examples of progression criteria as part of the discovery process for neglected diseases have been described by Nwaka et al. ${ }^{20}$ The major decision points are listed below and shown in Figure 2.
- Hit selection: The decision to proceed to in vivo studies and hit expansion based on the identification of a single compound from library screening or literature search.

- Lead selection: The decision to proceed to chemical optimization based on review of a series of chemical analogues of the hit compound.

- Optimized lead selection: The decision to proceed to regulatory preclinical toxicology (GLP [Good Laboratory Practice] Toxicology) and GMP (Good Manufacturing Practice) pharmaceutical development of one compound or a small number of compounds from the same chemical class.

- Drug candidate selection: The decision to proceed to clinical evaluation of one compound or a small number of compounds from the same chemical class.

In addition, key activities and partners are listed for each stage of drug discovery to ensure that sufficient resources are allocated to the program and that research progresses in the shortest timeframe as activities are conducted in parallel wherever possible. At each decision point, the information may result in a go/no go decision. In some instances, it may highlight additional research required before fully committing to the next stage of drug discovery.

The key activities for each decision point and the expected outcomes for these activities are defined to guarantee that continued development will address the expectations defined by the TPP.

\section{Decision matrices}

The decision matrix is an operational tool designed to support the drug discovery manual. In addition to the key assays

Table I Target product profile for developing combinations from existing VL treatments and NCEs

\begin{tabular}{|c|c|c|}
\hline & For VL combinations of existing VL treatments & For VL NCEs \\
\hline Target label & $\mathrm{VL}$ & $V^{a}$ \\
\hline Species & All species & All species \\
\hline Distribution & All areas & All areas \\
\hline Target population & $\begin{array}{l}\text { Immunocompetent and immunosuppressed, } \\
\text { adults and children }\end{array}$ & $\begin{array}{l}\text { Immunocompetent and immunosuppressed, } \\
\text { adults and children }\end{array}$ \\
\hline Clinical efficacy & $>95 \%$ (phase 3$)$ & $>95 \%$ \\
\hline Resistance & Active against resistant strains & Active against resistant strains \\
\hline Safety and tolerability & No AEs requiring patient monitoring & No AEs requiring monitoring \\
\hline Contraindications & None/few as defined by current drugs & None \\
\hline Interactions & & None - compatible for combination therapy \\
\hline Formulation & A mix of iv, im \pm oral & Oral/im depot \\
\hline Treatment regimen & $\leq 10$ day treatment & I/day for 10 days po/3 shots im over 10 days \\
\hline Feasibility & $\begin{array}{l}\text { Most of treatment given as outpatient } \\
\text { (eg, oral treatment) }\end{array}$ & Outpatient management with minimal follow-up \\
\hline Stability & As defined by current drugs & 3 years in zone IV \\
\hline Cost & $\leq$ US\$75/course & $<$ US\$I0/course \\
\hline
\end{tabular}

Notes: aNCE should ideally also target PKDL. TPPs are revised on a regular basis.

Abbreviations: AE, adverse event; iv, intravenous; im, intramuscular; NCE, new chemical entity; PKDL, post-kala-azar dermal leishmaniasis; po, orally; VL, visceral leishmaniasis. 


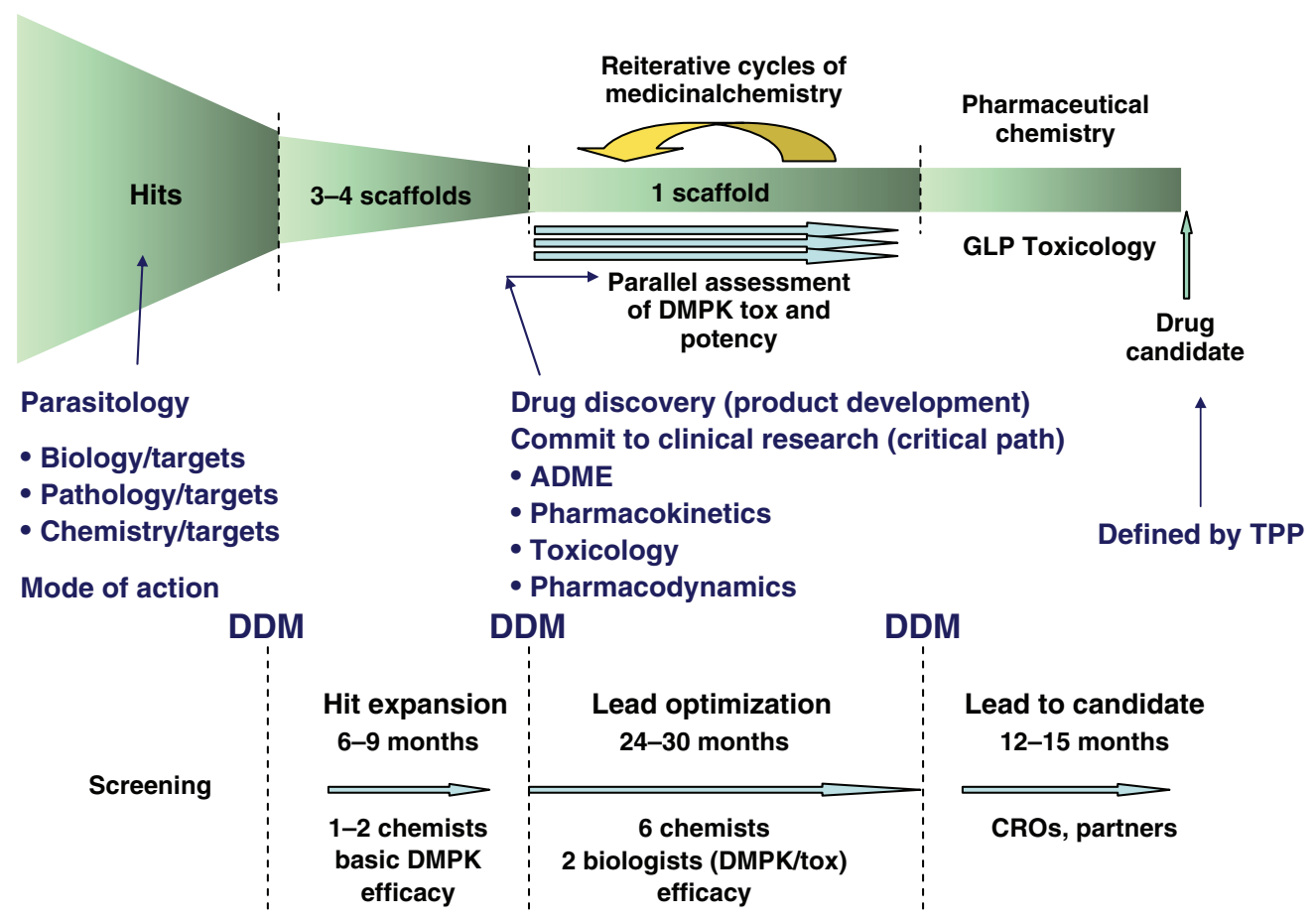

Figure 2 DNDi's drug discovery process with decision-making tools and key decision points.

Abbreviations: ADME, absorbtion-distribution-metabolison-excretion; DDM, drug discovery manual; TPP, target product profile; DMPK, distribution-metabolison-pharmacecotinetic.

specified for the decision points, simpler surrogate assays are listed to enable more rapid screening of molecules that are synthesized as part of medicinal chemistry programs. A simple spreadsheet, in which values for each new compound are entered, allows for ready comparison with ideal and acceptable values. By color coding the cells on the basis of the value entered, it is possible to rapidly assess progress during the synthesis of a particular chemical series.

In addition, it is recommended that representative compounds from the main classes with defined biological activity should be regularly tested to assess the true predictive value of the surrogate assays.

\section{Data management}

Regular and spontaneous communication between all members of a research team is essential for efficient progression of a lead optimization program. In most cases, not all members are located at the same site.

To provide all participants ready access to generated data, DND $i$ uses a proprietary Web-based data management system. All researchers enter data in templates that have been formatted to suit the data they generate, either chemical or biological. The data are structure and substructure searchable, and every researcher can review all data within the context of their specific expertise. A Web-based portal can store files in various formats and is used for circulation of reports and for storing complex supporting data and background information such as papers and patents.

\section{Expert committees}

$\mathrm{DND} i$ works with consultants who are expert in different disciplines to provide ongoing critical review of the programs. The programs are also regularly reviewed by the Scientific Advisory Committee of DND $i$ which is formed by prominent scientists with expertise in various scientific disciplines.

\section{Achievements and next challenges}

The PDP for NTD drug development relies on strong partnerships between industry and academia, and on sharing of knowledge that is often held as proprietary by different partners. In addition to the application of best research practices (eg, TPPs, drug discovery manuals, critical path), building of trust and open communication are crucial to success. Although the concept of the PDP is still in its infancy, many productive alliances have been built. For example, DND $i$-coordinated Fixed-Dose Artesunate-Containing Therapy (FACT) Project Consortium was developed and made available two new improved treatments for malaria. In 2007, DND $i$ in collaboration with sanofi-aventis, registered its first product, the antimalarial artesunate-amodiaquine fixed-dose combination (ASAQ), by applying its innovative R\&D model for the treatment of noncomplicated Plasmodium falciparum 
malaria. In 2008, DND $i$ in partnership with a public institution (Farmanguinhos, Brazil) launched its second product for malaria, artesunate-mefloquine fixed-dose combination (ASMQ) in Brazil using a similar model. In 2009, following the results of a Phase 3 clinical trial testing the efficacy of a combination treatment of nifurtimox and eflornithine (NECT) against stage 2 HAT, nifurtimox was added to WHO Essential Medicine List for the treatment of HAT. NECT is recommended by WHO for the treatment of HAT.

More importantly, candidates issued from DND $i$ discovery pipeline are now starting to move forward towards preclinical and clinical development, indicating the emerging success of the strategy of the PDP model for NTD drug discovery and development.

There is, however, a need to carry on strengthening our existing preclinical pipeline to cope with the attrition rate inherent to any R\&D activity. The identification of novel lead series and clinical candidates supported by strong backup programs is indeed key to ensure a timely delivery of new treatments for patients suffering from neglected diseases.

Finally, awareness towards those affected by NTDs still needs to be increased. We indeed strongly believe it is only by raising awareness that we can ensure that truly modern treatments (safe, efficacious, easy to administer) and funding will be made available.

\section{Acknowledgments}

The authors would like to thank Federica Giovannini and Rob Don for critical reading of the manuscript.

DND $i$ is grateful to its donors, public and private, who have provided funding to DND $i$ since its inception in 2003. With the support of these donors, DND $i$ is well on its way to achieving the objectives of a robust pipeline with the aim to deliver $6-8$ new treatments by 2014. A full list of DNDi's donors can be found at http://www.dndi.org/index.php/donors.html?ids=8.

The donors had no role in study design, data collection and analysis, decision to publish, or preparation of the manuscript.

For more information about DND $i$ see http://www.dndi.org.

\section{Disclosure}

The authors report no conflicts of interest in this work.

Drug Design, Development and Therapy

\section{Publish your work in this journal}

Drug Design, Development and Therapy is an international, peerreviewed open-access journal that spans the spectrum of drug design and development through to clinical applications. Clinical outcomes, patient safety, and programs for the development and effective, safe, and sustained use of medicines are a feature of the journal, which

\section{References}

1. Feasey N, Wansbrough-Jones M, Mabey DC, Solomon AW. Neglected tropical diseases. Br Med Bull. 2010;93:179-200.

2. Hotez PJ, Kamath A. Neglected tropical diseases in sub-saharan Africa: review of their prevalence, distribution, and disease burden. PLoS Negl Trop Dis. 2009;3:e412.

3. Hotez PJ, Pécoul B. "Manifesto" for advancing the control and elimination of neglected tropical diseases. PLoS Negl Trop Dis. 2010; 4:e718.

4. World Health Organization. http://www.who.int/topics/tropical_ diseases/en/. Accessed December 6, 2010.

5. Conteh L, Engels T, Molyneux DH. Socioeconomic aspects of neglected tropical diseases. Lancet. 2010;375:239-247.

6. Mathers CD, Ezzati M, Lopez AD. Measuring the burden of neglected tropical diseases: the global burden of disease framework. PLoS Negl Trop Dis. 2007;1:e114.

7. Chirac P, Torreele E. Proportion of new drugs developed over the period from 1975 to 2004 that were for neglected tropical diseases and tuberculosis. Lancet. 2006;12:1560-561.

8. World Health Organization/Industry Drug Development Working Group. http://www.who.int/intellectualproperty/documents/en/IFPMA. pdf. Accessed December 6, 2010.

9. Moran M. A breakthrough in R\&D for neglected diseases: new ways to get the drugs we need. PLoS Med. 2005;2:e309.

10. Nwaka S, Ridley RG. Virtual deug discovery and development for neglected diseases through public-private partnerships. Nat Rev Drug Disc. 2003;2:919-928.

11. Croft SL. Public-private partnership: from there to here. Trans $R$ Soc Trop Med Hyg. 2005;99 Suppl 1:S9-S14.

12. The George Institute for International Health. G-FINDER: "Global Funding of Innovation for Neglected Disease" survey. Available from: http://www.thegeorgeinstitute.com. Accessed November 15, 2010.

13. Moran M, Guzman J, Ropars AL, et al. Neglected disease research and development: how much are we really spending? PLoS Med. 2009;6: e1000030.

14. Pécoul B. New drugs for neglected diseases: from pipeline to patients. PLoS Med. 2004;1:e6.

15. Torreele E, Bourdin B, Tweats D, et al. Fexinidazole - a new oral nitroimidazole drug candidate entering clinical development for the treatment of sleeping sickness. PLoS Negl Trop Dis. 2010;4(12):e923.

16. Preziosi P. Attrition rate R\&D of pharmaceutical drugs. Nat Rev Drug Disc. 2004;3:521-526.

17. Woosley RL, Cossman J. Drug development and the FDA's critical path initiative. Clin Pharmacol Rev. 2007;81:129-133.

18. Don R, Chatelain E. Drug discovery for neglected diseases: view of a public-private partnership. In: Selzer PM, editor. Antiparasitic and Antibacterial Drug Discovery: From Molecular Targets to Drug Candidates. Weinheim, Germany: Wiley-VCH GmbH \&Co. KGaA; 2009: 33-43.

19. Curry S, Brown R. The target product profile as a planning tool in drug discovery research. Pharmatech. 2003:67-71.

20. Nwaka S, Ramirez B, Brun R, et al. Advancing drug innovation for neglected disease - criteria for lead progression. PLoS Negl Trop Dis. 2009;3:e440.

Submit your manuscript here: http://www.dovepress.com/drug-design-development-and-therapy-journal 